\title{
CIF Applications. VIII. pdb2cif: translating PDB entries into mmCIF format $\dagger$
}

\author{
Herbert J. Bernstein, ${ }^{a}$ Frances C. Bernstein ${ }^{b}$ and Philip E. Bourne ${ }^{c, d}$ at ${ }^{a}$ Bernstein + Sons, 5 Brewster Lane, Bellport, $N Y$ \\ 11713-2803, USA, ${ }^{b}$ Biology Department, Brookhaven National Laboratory, Upton, NY 11973-5000, USA, 'San Diego \\ Supercomputer Center, PO Box 85608, San Diego, CA 92186-9784, USA, and "Department of Pharmacology, University of \\ California, San Diego, CA 92093-0365, USA. E-mail: yaya@bernstein-plus-sons.com
}

(Received 8 May 1997; accepted 14 May 1997)

\begin{abstract}
pdb2cif is a new version of an awk script originally written by P. E. Bourne in 1993 to translate from the 1992 Protein Data Bank (PDB) format to the then-emerging macromolecular Crystallographic Information File (mmCIF) definition. This new version of pdb2cif translates from all current PDB formats, including the 1992 PDB format and the 1996 PDB Atomic Coordinate Entry Format, Version 2.0, to the 1997 mmCIF format as defined in the mmCIF dictionary 1.0.00. The program is provided as an $\mathrm{m} 4$ script from which both perl and awk versions can be produced. The program identifies mmCIF entities implicitly by sequence homology among PDB SEQRES records. With minor additions to the dictionary, the resultant mmCIF data-sets are substantially compliant with the mmCIF 1.0 .00 dictionary.
\end{abstract}

\section{Introduction}

The program pdb2cif reads entries in Protein Data Bank (PDB) format (Bernstein et al., 1977) or PDB Atomic Coordinate Entry format (Protein Data Bank, 1996) and converts them to macromolecular Crystallographic Information File (mmCIF) format (Fitzgerald et al., 1996; Bourne et al., 1997). All valid $\mathrm{PDB}$ record types are converted, but most PDB REMARK records are carried forward as text, rather than being parsed any further. The resulting entries are substantially compliant with dictionary definition language version 2 (DDL2) (Berman \& Westbrook, 1994; Westbrook \& Hall, $1995)$ and mmCIF rules with the addition of a small number of new token definitions.

The Protein Data Bank format has been used for over 20 years to archive macromolecular data, is produced by many refinement programs and is used as an input format by many applications. The adoption of the mmCIF dictionary (Fitzgerald et al., 1996) by the IUCr, in response to the need to represent explicitly a larger amount of data that can be parsed by computer (necessary as the number of structures continues to grow exponentially), has made translation from PDB format to mmCIF format a pressing issue.

In this paper we review the techniques used in pdb2cif to move from structures represented in PDB format to $\mathrm{mmCIF}$ format. Some data items have direct mapping with minor syntactic adjustment, such as for author names and journal references. Other data items, however, require us to recast our thinking along new lines. For example, the PDB format works

† This paper is one of a series on CIF applications. Offprints are available from The Managing Editor, International Union of Crystallography, 5 Abbey Square, Chester CH1 2HU, England. See text of paper for availability of program(s) by e-mail. with chains and heterogen groups, while mmCIF uses entities (discrete chemical components). Proper identification of entities in a PDB entry may require looking for sequence homology. As another example, consider beta sheets. The PDB format treats a bifurcated sheet as two distinct sheets that happen to have certain strands in common, while mmCIF allows all the strands involved to be represented as a single sheet. This requires strand matching and alignment to go from PDB format to mmCIF. What has currently been automated in pdb2cif and what still requires human intervention is discussed.

\section{Outline of the PDB format}

The Protein Data Bank describes a macromolecular structure using a format containing records with fixed fields that are order dependent. In this context, a record is a line of text. The first six characters of each record contain a left-justified string of from three to six upper-case characters that specifies a particular PDB 'record type'. The record type implies the layout of the information in that record. For some record types fields of information may span multiple records of the same type. For many such record types, continuation is indicated by an integer in columns 9-10. In most cases, the meaning of the information found in specific columns of a record type is fixed and is specified in external documents rather than within the PDB entry itself. In most cases, all records of a given record type are grouped together. The order of presentation of different record types within an entry is fixed. In all cases the records are no more than 80 characters long and, in entries conforming to the PDB 1992 or earlier formats, there is no structural information past column 72 . There are several variations of the PDB format which have been used since its adoption. Table 1 is a composite of the 1992 and 1996 versions, since data-sets in both formats are still in use.

In the coordinate section, ATOM records are used for 'standard' residues and HETATM records are used for atoms in heterogens. For any given atom in a given conformation, the ordering of records is [ATOM|HETATM][SIGATM] [ANISOU][SIGUIJ] as a single group of records. Groups of records specifying the alternate conformations for the same atom follow immediately. These groups of records are then organized in an ordering determined by templates for standard residues or heterogens. Records in the coordinate section associated with a particular model in an NMR entry with multiple models are delimited by a MODEL/ENDMDL pair. Chains are terminated by TER records. Except within the coordinate section, all records for any given record type are grouped together. 
Table 1. Outline of the PDB data format

An entry comprises sections (bold) containing the record types (italic for record types in both 1992 and 1996 formats. plain text for record types introduced in 1996) listed in the following order. Optional record types are contained in square brackets. It should be pointed out that the records REMARK 3-999 have more internal structure in the 1996 format than in previous formats.

\begin{tabular}{|c|c|c|c|}
\hline \multicolumn{4}{|l|}{ Title section } \\
\hline$H E A D E R$ & {$[O B S L T E]$} & TITLE & [CAVEAT] \\
\hline COMPND & SOURCE & KEYWDS & {$[E X P D T A]$} \\
\hline AUTHOR & REVDAT & {$[S P R S D E]$} & {$[J R N L]$} \\
\hline REMARK 1 & REMARK 2 & REMARK 3 & [REMARK 4-999] \\
\hline \multicolumn{4}{|c|}{ Primary structure section } \\
\hline [MODRES] & DBREF & [SEQADV] & {$[S E Q R E S \mid$} \\
\hline \multicolumn{4}{|c|}{$\begin{array}{l}\text { Footnote section (1992 format only) } \\
\text { FTNOTE }\end{array}$} \\
\hline \multicolumn{3}{|c|}{ Heterogen section } & {$[F O R M U L]$} \\
\hline \multicolumn{4}{|c|}{ Secondary structure section } \\
\hline$[H E L I X]$ & {$[S H E E T]$} & {$[T U R N]$} & \\
\hline \multicolumn{4}{|c|}{ Connectivity annotation section } \\
\hline $\begin{array}{l}{[S S B O N D]} \\
{[\text { CISPEP] }}\end{array}$ & [LINK] & [HYDBND] & [SLTBRG] \\
\hline \multicolumn{4}{|c|}{$\begin{array}{l}\text { Miscellaneous features section } \\
{[S I T E]}\end{array}$} \\
\hline \multicolumn{4}{|c|}{ Crystallographic and coordinate transformation section } \\
\hline $\begin{array}{l}\text { CRYST1 } \\
{[T V E C T]}\end{array}$ & ORIGXn & SCAL.En & {$[M T R I X n]$} \\
\hline \multicolumn{4}{|c|}{ Coordinate section } \\
\hline$[M O D E L]$ & {$[$ ATOM $]$} & {$[S I G A T M]$} & {$[A N I S O U]$} \\
\hline$[S / G U I J]$ & {$[T E R]$} & {$[$ HETATM] } & {$[E N D M D L]$} \\
\hline \multicolumn{4}{|c|}{$\begin{array}{l}\text { Connectivity section } \\
{[\text { CONECT] }}\end{array}$} \\
\hline \multicolumn{4}{|c|}{ Bookkeeping section } \\
\hline MASTER & END & & \\
\hline
\end{tabular}

Fig. 1 shows part of the coordinate section from the PDB entry 4INS (pig insulin) (Baker et al., 1988) in the format in use in 1989 and how the same information would be presented in the 1996 format. Note that columns $78-79$ now contain the right-justified element symbol in the 1996 format.

\section{Outline of $\mathbf{~ m m C I F}$}

The new mmCIF format is one of a family of STAR (Selfdefining Text Archive and Retrieval) file formats which uses a tag-value style of presentation and has very little sensitivity to the ordering of the information (Hall, 1991: Hall \& Spadaccini, 1994). Since no fixed positions for fields are defined in mmCIF, the format of mmCIF data-sets (Fitzgerald et al., 1996; Bourne et al., 1997) is much less rigidly defined than is the case for fixed-field formats such as the PDB format. Information is presented either in tag-value pairs or in column-headed tabular form. Tags are distinguished from values by an initial underscore. Information is constrained to 80-column lines, but spacing between fields is arbitrary. In mmCIF, tags are organized into category groups and categories. Individual tag-value pairs from different categories may be placed anywhere within a data-set, but it is considered good practice to group the tag-value pairs from a given category together. When the STAR construct 'loop_' is used to introduce a table, all the data items within that table must have tags from the same category and all the data items for that category for which any information is being presented should be placed in the same table. The category is a name for the table. The category groups and associated categories defined in the mmCIF dictionary are given in Table 2.

Each category contains multiple tags. The name of each tag begins with its category followed by a period. In STAR, a table of information is created by the special token 'loop_' followed by the tags that head the columns of the table, followed by the rows of values. If a table is given at all, certain tags are mandatory and certain values cannot be missing. Each row of a table must have a unique key, consisting of the values of certain designated columns within that row. In addition, some information is mandatory in mmCIF data-sets to ensure a complete coherent presentation of information

1989 format:

\begin{tabular}{|c|c|c|c|c|c|c|c|c|c|c|c|c|c|}
\hline ATOM & 1 & $\mathbf{N}$ & GLY & A & 1 & -8.863 & 16.944 & 14.289 & 1.00 & 21.88 & 1 & 4 INS & 235 \\
\hline ATOM & 2 & CA & GLY & A & 1 & -9.929 & 17.026 & 13.244 & 1.00 & 22.85 & 1 & 4 INS & 236 \\
\hline ATOM & 3 & $c$ & GLY & A & 1 & -10.051 & 15.625 & 12.618 & 1.00 & 43.92 & 1 & 4 INS & 237 \\
\hline АTOM & 4 & 0 & GLY & A & 1 & -9.782 & 14.728 & 13.407 & 1.00 & 25.22 & 1 & 4 INS & 238 \\
\hline ATOM & 5 & $\mathbf{N}$ & ILE & A & 2 & -10.333 & 15.531 & 11.332 & 1.00 & 26.28 & 1 & 4INS & 239 \\
\hline АТОM & 6 & CA & ILE & A & 2 & -10.488 & 14.266 & 10.600 & 1.00 & 20.84 & 1 & 4INS & 240 \\
\hline АТОM & 7 & C & ILE & A & 2 & -9.367 & 13.302 & 10.658 & 1.00 & 11.81 & 1 & 4INS & 241 \\
\hline АTOM & 8 & 0 & ILE & A & 2 & -9.580 & 12.092 & 10.969 & 1.00 & 20.31 & 1 & 4INS & 242 \\
\hline ATOM & 9 & $\mathrm{CB}$ & ILE & $\mathbf{A}$ & 2 & -10.883 & 14.493 & 9.095 & 1.00 & 40.00 & 1 & 4INS & 243 \\
\hline ATOM & 10 & CG1 & ILE & A & 2 & -11.579 & 13.146 & 8.697 & 1.00 & 36.74 & 1 & 4INS & 244 \\
\hline
\end{tabular}

1996 format:

$\begin{array}{llll}\text { ATOM } & 1 & \text { N } & \text { GLY A } \\ \text { ATOM } & 2 & \text { CA } & \text { GLY A } \\ \text { ATOM } & 3 & \text { C } & \text { GLY A } \\ \text { ATOM } & 4 & \text { O } & \text { GLY A } \\ \text { ATOM } & 5 & \text { N } & \text { ILE A } \\ \text { ATOM } & 6 & \text { CA } & \text { ILE A } \\ \text { ATOM } & 7 & \text { C } & \text { ILE A } \\ \text { ATOM } & 8 & 0 & \text { ILE A } \\ \text { ATOM } & 9 & \text { CB } & \text { ILE A } \\ \text { ATOM } & 10 & \text { CG1 ILE A }\end{array}$

$\begin{array}{rrrrrr}-8.863 & 16.944 & 14.289 & 1.00 & 21.88 \\ -9.929 & 17.026 & 13.244 & 1.00 & 22.85 \\ -10.051 & 15.625 & 12.618 & 1.00 & 43.92 & 1 \\ -9.782 & 14.728 & 13.407 & 1.00 & 25.22 \\ -10.333 & 15.531 & 11.332 & 1.00 & 26.28 & 1.00 \\ -10.488 & 14.266 & 10.600 & 1.00 & 20.84 & 100 \\ -9.367 & 13.302 & 10.658 & 1.00 & 11.81 & 1 \\ -9.580 & 12.092 & 10.969 & 1.00 & 20.31 \\ -10.883 & 14.493 & 9.095 & 1.00 & 40.00 \\ -11.579 & 13.146 & 8.697 & 1.00 & 36.74\end{array}$

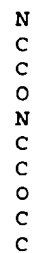

Fig. 1. Example of the coordinate section from PDB entry 4INS in the format in use in 1989 and in the 1996 format. 
Table 2. Category groups (bold) and categories in the mmCIF dictionary (version 1.0.00)

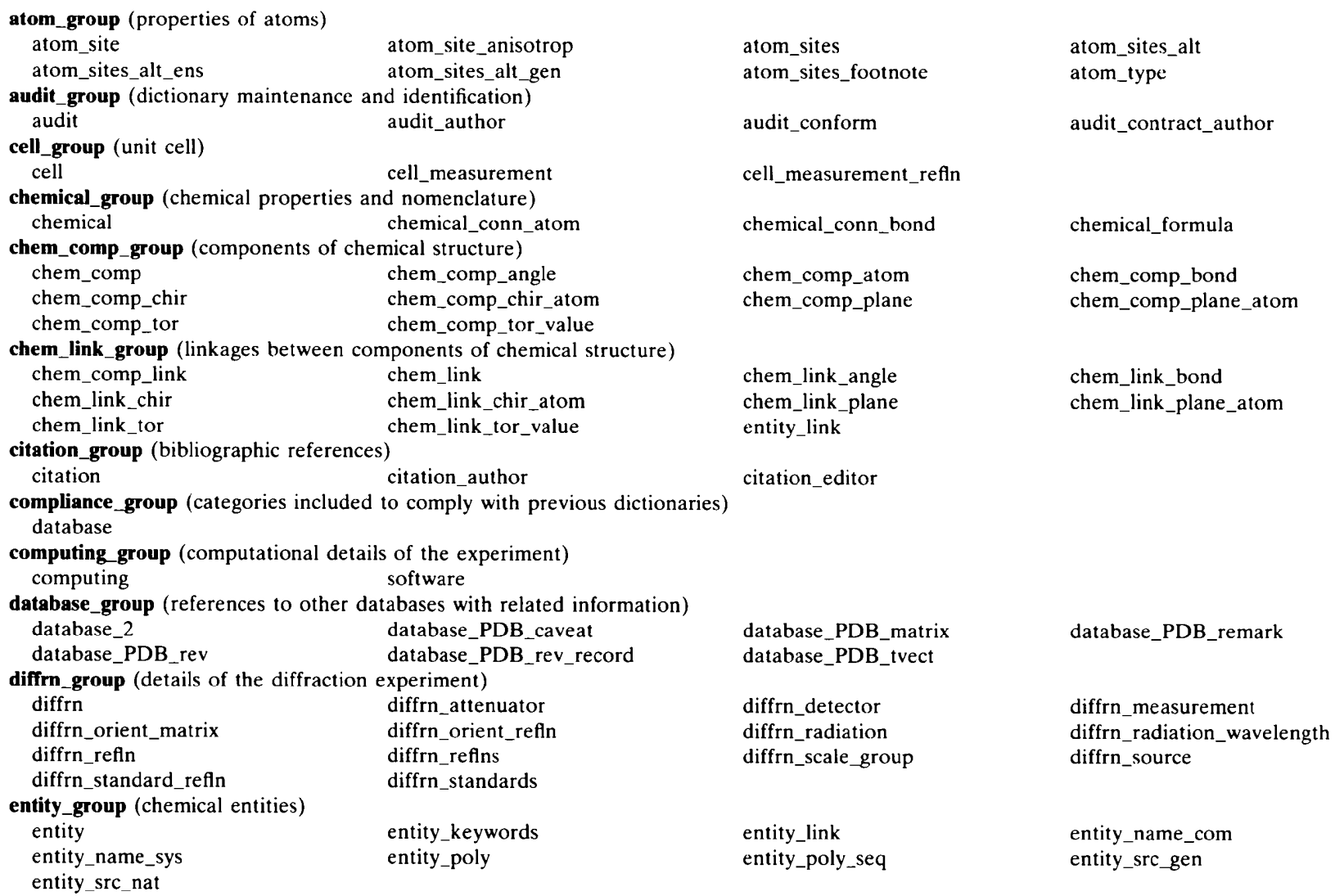

about a macromolecular structure. Such additional mandatory tags in a table need not have distinct values row to row. For example, in the atom_site category, the key is the data item _atom_site.id, which uniquely identifies each row in the atom_site table. Values for_atom_site.type_symbol (e.g. $\mathrm{C}, \mathrm{N}, \mathrm{O}$ ) are also mandatory, but, naturally, they are not unique in each row.

In mmCIF format, once the tag heading a column is given, values must be given for that column in every row. When the information to be given is not known, a question mark is used in place of the required value. When a value is otherwise intentionally not given, a period is used in place of the required value. In translating from $\mathrm{PDB}$ format to $\mathrm{mmCIF}$, it is often necessary to recognize blank fields in PDB records and to find a value to use in the equivalent mmCIF table. With some exceptions noted below, a period is an appropriate equivalent to the PDB blank.

Fig. 2 gives an extract from an mmCIF conversion of $\mathrm{PDB}$ entry 4INS showing the beginning of the table giving the tags and values in the atom_site category. Because tags are always given, the same information can be presented in different orderings. Note that the mmCIF format does not depend on the columns shown in Fig. 2, just on a consistent ordering of tags versus data values. Also note that a period had to be given in each row as a place-holder for the unspecified values of _atom_site.label_alt_id. The period is a 'metacharacter' in mmCIF denoting an unspecified value. A question mark, which has the slightly different meaning of a missing value, could also have been used.

\section{Relationship between mmCIF and PDB format}

The relationship between mmCIF and PDB format is complex. There are differences both in syntax and in content. These differences are summarized in Table 3.

Handling the syntactic differences between PDB format and $\mathrm{mmCIF}$ format involves attention to detailed information relating various $\mathrm{PDB}$ fields to appropriate mmCIF tags and is a straightforward translation using specific rules. However, handling the differences in content requires much more from a translation program. Translation of PDB polypeptide and polynucleotide chains into $\mathrm{mmCIF}$ chemical entities is a case in point. While nonpolymeric heterogens are assigned an explicit 'component number' in PDB format, which is essentially equivalent to an mmCIF_entity.id, more analysis is needed when dealing with chains. In general, the most difficult issues arise from the concept of 'normalization' (see below). Other areas are less troublesome. PDB and mmCIF formats agree simply and directly for some data items, such as cell parameters, and permit a simple tabular mapping, as shown in Fig. 3, by an extract from the concordance which is available as part of the pdb2cif program release. Other important 
Table 2. (cont.)

entry_group (the entire data block) entry entry_link

exptl_group (details of the experimental conditions)

exptl exptl_crystal

exptl_crystal_grow_comp

exptl_crystal exptl_crystal_face

exptl_crystal_grow

geom_group (internal coordinates)

geom

geom_angle

geom_bond

geom_contact

eom_hbond

geom_torsion

iucr_group (internal processing and manuscript submission by the IUCr staff)

journal publ_body

journal_index

publ_manuscript_incl

publ

pdb_group (pertaining to PDB file format or data processing codes, overlaps much of database_group)

database_PDB_caveat database_PDB_matrix database_PDB_remark

database_PDB_rev_record database_PDB_tvect

phasing_group (phasing)

phasing

phasing_MAD_clust

phasing_mir

phasing_MIR_der_site

refine_group (describe refinement)

refine

refine_ls_restr

phasing_averaging

phasing_MAD_expt

phasing_MIR_der

phasing_MIR_shell

refine_analyze

refine_Is_restr_ncs

refln_group (details of reflection measurements)

refln reflns

struct_group (crystallographic structure)

struct

struct_biol_keywords

struct conn

struct_mon_nucl

struct_ncs_dom_lim

struct_ref

struct_sheet_hbond

struct_asym

struct_biol_view

struct_conn_type

struct_mon_prot

struct_ncs_ens

struct_ref_seq

struct_site

struct_sheet_order

struct_site_gen

symmetry_group (symmetry information)

symmetry

symmetry_equiv phasing_isomorphous

phasing_MAD_ratio

phasing_MIR_der_refln

phasing_set

refine_B_iso

refine_ls_shell

reflns_scale

struct_biol

struct_conf

struct_keywords

struct_mon_prot_cis

struct_ncs_ens_gen

struct_ref_seq_dif

struct_sheet_range

struct_site_keywords publ_author

database_PDB_rev

phasing_mad

phasing_MAD_set

phasing_MIR_der_shell

phasing_set_refin

refine_hist

refine_occupany

reflns_shell

struct_biol_gen

struct_conf_type

struct_mon_details

struct_ncs_dom

struct_ncs_oper

struct_sheet

struct_sheet_topology

struct_site_view macromolecular data descriptors, because of the very different views of the same data, require complex transformations.

For example, beta sheets are built from beta strands. In mmCIF, all the strands in all shects are listed in one struct_sheet_range table. The relative ordering and oricntation of all strands in all sheets are given in one struct_sheet_order table. The hydrogen bonding among all strands in all sheets is listed in one struct_sheet_hbond

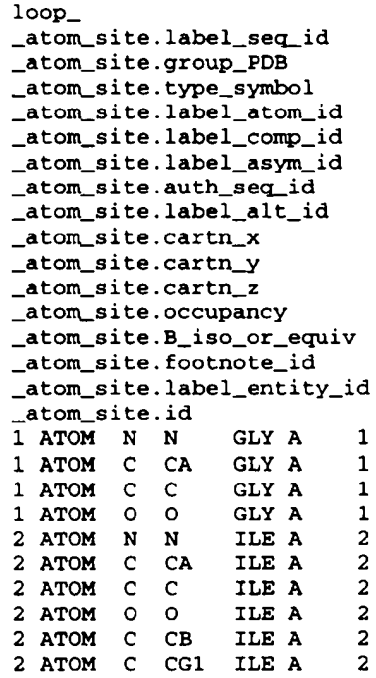

Fig. 2. Beginning of the atom_site table for mmCIF conversion of PDB entry 4INS. 
Table 3. Major differences in syntax and content between $P D B$ format and mmCIF format

Syntax

mmCIF

Tag-value definitions

Little order dependence

Strict table structure

Upper/lower case

yyyy-mm-dd dates

Family-name-first author names

Related items may have to appear in separate tables

\section{$\overline{P D B}$}

Fixed fields

Strong order dependence

Some information nontabular

Upper case only

dd-mmm-yy dates (dd-mmm-

yyyy in some REMARKS)

Family-name-last author names

PDB
Less normalization
Structures defined using
chains and heterogen
groups

is database oriented. This causes information from PDB records such as SHEET or JRNL to be distributed across multiple mmCIF categories and information from separate PDB records to be gathered into common mmCIF tables. For example, the PDB-record-to-mmCIF-category mapping of the primary structure section used in pdb2cif is shown in Fig. 6.

One last issue that arises in conversion from PDB format to mmCIF is selection of an ordering of the information in an $\mathrm{mmCIF}$ data-set. There is no required ordering. One common practice is to order tag-value pairs and tables alphabetically, but this places the table of atomic coordinates in the atom_site category first, placing a large block of information before categories that identify the data-set. For readability, it is helpful to place information from the _entry.id, _struct.title, the contents of the struct_keywords, audit_author, citation, citation_editor, citation_author, rens, database_PDB_remark, cell, symmetry, audit, entity_poly_seq, entity, struct_asym, chem_comp, database_PDB_matrix, atom_sites and atom_sites_footnote categories before the atom_site table. We follow this practice in pdb2cif.

\section{The program pdb2cif}

table. The general characteristics of all sheets per se is given in one struct_sheet table. In PDB format, sheets are described by one set of sheet records for each simple, nonbifurcated sheet. To convert from PDB format to mmCIF format, a list of all strands must be extracted from the SHEET records, sorted to remove duplicates, and the information placed in a struct_sheet_range table. All strand-to-strand relationships are extracted and placed in a struct_sheet_order table, etc. A diagram of PDB entry $2 \mathrm{ACE}$ (native acetylcholinesterase) (Raves et al., 1997) is given in Fig. 4 showing the strands forming sheets. Other secondary structure is not shown. A small sheet of three strands is on the top left and a larger sheet of 11 strands is on the right. Residue 16 is common to both sheets. The SHEET information from this PDB entry is given in Fig. 5(a). The same information converted to mmCIF format by $p d b 2 c i f$ is given in Figs. 5(b) and 5(c).

The scattering of information from a PDB SHEET record type into various tables is an example of 'normalization' (Codd, 1970, 1972). Normalization is a concept from the design of databases in which data are organized into the rows and columns of tables with a single data item in each table position, with unique keys to identify each row, and minimal repetition of the same information, so that it is easier to update, check and retrieve data reliably. Although not developed explicitly for such database considerations, mmCIF pdb2cif converts PDB entries into mmCIF data-sets. (The tion presented as a single document describing some set of data. At present, each data-set produced by mmCIF contains one CIF data_block, even if multiple NMR models are described.) Most, but not all, common PDB record types are converted. The exceptions are the new structured PDB REMARK records introduced in April 1996 (Protein Data Bank, 1996), which, as of this writing, are still evolving. These REMARK records are preserved as text associated with the database_PDB_remark.text tag, rather than being parsed internally to provide values for tags in other categories. The program also cannot resolve some of the ambiguities involved in the analysis of the new keyword fields for the PDB COMPND and SOURCE records and treats those as text as well. The program has gone through extensive changes since 1993 as both mmCIF and the PDB format have evolved. The program, which was initially written as an awk script, is now available as an $\mathrm{m} 4$ (Kernighan \& Ritchie, 1977) macro document that produces either perl or awk versions. The perl version is recommended.

The pdb2cif.m4 macro document contains approximately 6500 lines of text, which generates a similarly sized awk script or over 10000 lines of perl code (due to in-lining of certain critical functions). On modern processors with sufficient memory ( 32 to 64 Mbytes of available RAM), the conversion term 'data-set' refers to the comments and mmCIF informa-

$\begin{array}{llll}\text { PDB Field } & \text { Content } & \text { Type of Transformation } & \text { Related manCIF field } \\ \text { CRYST1[1-6] } & \text { CRYST1 } & \text { NA } & \\ \text { CRYST1[7-15] } & \text { a } & \text { equivalent to } & \text {-cell.length_a } \\ \text { CRYST1[16-24] } & \text { b } & \text { equivalent to } & \text {-cell.length_b } \\ \text { CRYST1[25-33] } & \text { C } & \text { equivalent to } & \text {-cell. length_C } \\ \text { CRYST1[34-40] } & \text { alpha } & \text { equivalent to } & \text {-cell.angle_alpha } \\ \text { CRYST1[41-47] } & \text { beta } & \text { equivalent to } & \text {-cell.angle_beta } \\ \text { CRYST1[48-54] } & \text { gamma } & \text { equivalent to } & \text {-cell.angle_gamma } \\ \text { CRYST1[56-66] } & \text { SGroup } & \text { equivalent to } & \text {-symetry.space_group_name_H-M } \\ \text { CRYST1[67-70] } & \text { Z } & \text { equivalent to } & \text {-cell.2_PDB }\end{array}$

Fig. 3. Example of a simple concordance between the PDB CRYST1 record type and mmCIF format. 


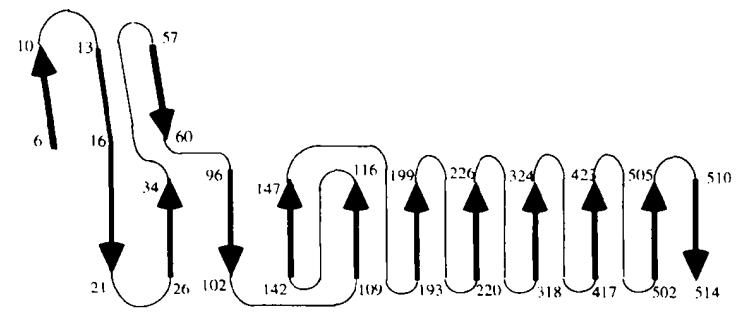

Fig. 4. Diagram of PDB entry 2ACE showing strands forming sheets. of a PDB entry to an mmCIF data-set takes from several seconds to a few minutes depending on the size of the PDB entry. The longest processing times are, for example, in NMR entries with multiple models. The mmCIF data-sets produced are approximately the same size as the original PDB entries. Table 4 provides the statistics for some conversions done on an SGI R8000 Power Indigo-2 Extreme with 128 Mbytes of memory.

The time is approximately linear in the file size and dominated by the processing time of the atom list. The times given in Table 4 are wall-clock times and approximate the processor (a)

$\begin{array}{lrll}\text { SHEET } & 1 & \text { A } 3 & \text { LEU } \\ \text { SHEET } & 2 & \text { A } 3 & \text { GLY } \\ \text { SHEET } & 3 & \text { A } 3 & \text { VAL } \\ \text { SHEET } & 1 & \text { B11 } & \text { MET } \\ \text { SHEET } & 2 & \text { B11 } & \text { HIS } \\ \text { SHEET } & 3 & \text { B11 } & \text { TYR } \\ \text { SHEET } & 4 & \text { B11 } & \text { VAL } \\ \text { SHEET } & 5 & \text { B11 } & \text { THR } \\ \text { SHEET } & 6 & \text { B11 } & \text { THR } \\ \text { SHEET } & 7 & \text { B11 } & \text { ARG } \\ \text { SHEET } & 8 & \text { B11 } & \text { GLN } \\ \text { SHEET } & 9 & \text { B11 } & \text { GLY } \\ \text { SHEET } & 10 & \text { B11 } & \text { PHE } \\ \text { SHEET } & 11 & \text { B11 } & \text { MET }\end{array}$

$\begin{aligned} 6 & \text { THR } \\ 13 & \text { MET } \\ 57 & \text { ALA } \\ 16 & \text { PRO } \\ 26 & \text { PRO } \\ 96 & \text { PRO } \\ 142 & \text { SER } \\ 109 & \text { TYR } \\ 193 & \text { GLU } \\ 220 & \text { SER } \\ 318 & \text { ASN } \\ 417 & \text { PHE } \\ 502 & \text { LEU } \\ 510 & \text { GLN }\end{aligned}$

100

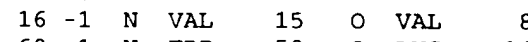

$\begin{array}{llllllll}60 & 1 & N & T R P & 58 & O & \text { LYS } & 14\end{array}$

210

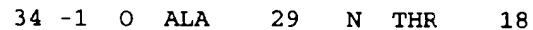

$102-1 \quad \mathrm{~N}$ ILE 99 O PHE 30

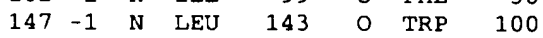

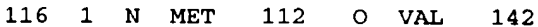

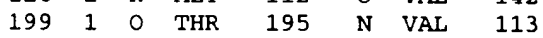

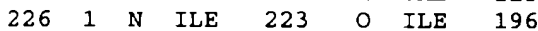

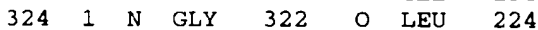

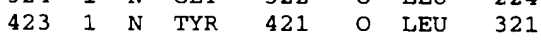

$5051 \mathrm{~N}$ ILE 503 O LEU 420

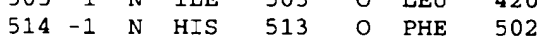

(b) loop

_struct_sheet.id

_struct_sheet.number_strands

A 3

B

_struct_sheet_hbond.sheet_id

-struct_sheet_hbond.sheet_id

struct_sheet_hbond.range_id_1
-struct_sheet_hbond.range_id_2

_struct_sheet_hbond.range_1_beg_auth_seq_id

_struct_sheet_hbond.range_1_beg_label_atom_id

_struct_sheet_hbond.range_2_beg_auth_seq_id

_struct_sheet_hbond.range_2_beg_label_atom_id

-struct_sheet_hbond.range_1_end_auth_seq_id

_struct_sheet_hbond.range_1_end_label_atom_id

_struct_sheet_hbond.range_2_end_auth_seq_id

_struct_sheet_hbond.range_2_end_label_atom_id

-struct_sheet_hbond.range_1_beg_label_seq_id

_struct_sheet_hbond.range_2_beg_label_seq_id

_struct_sheet_hbond.range_1_end_label_seq_id

_struct_sheet_hbond.range_2_end_label_seq_id

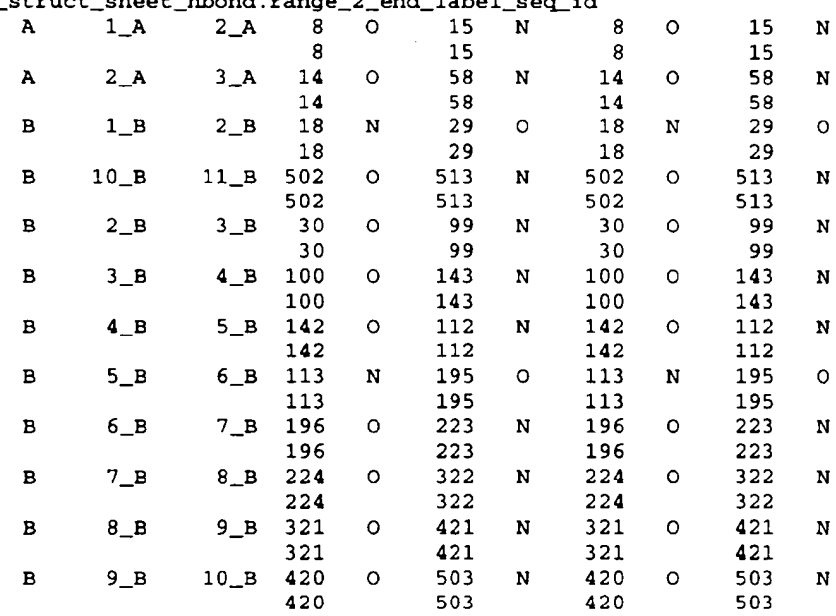

Fig. 5. (a) SHEET information from PDB entry 2ACE. (b) mmCIF struct_sheet and struct_sheet_hbond tables converted by $p d b 2 c i f$ from SHEET information in PDB entry 2ACE. $(c)$ mmCIF struct_sheet_order and struct_sheet_range tables converted hy $p d h 2 c i f$ from SHEET information in PDB entry $2 \mathrm{ACE}$. 
time on larger machines (assuming exclusive use). For large NMR entries processed on small machines, the wall-clock time can become very large due to extensive page swapping for the arrays used to hold the atom list.

The program produces summary warnings as comments at the end of each mmCIF data-set it produces. If a record is found with an unrecognized PDB record type it is reported in the AUDIT category. Warnings and converted records should be examined carefully, especially for the following record types.

COMPND, SOURCE, TITLE and CAVEAT are merged into _struct.title without further parsing. Additional information could be derived from PDB entries that follow the PDB 1996 format description when sufficient information for mapping of the PDB MOL_ID to mmCIF entities is available.

EXPDTA records use values that do not have a direct mapping to enumerated values for_exptl.method.

(c) loop

\begin{tabular}{|c|c|c|c|c|}
\hline \multicolumn{5}{|c|}{$\begin{array}{l}\text {-struct_sheet_order.sheet_id } \\
\text {-struct_sheet_order.range_id_1 } \\
\text {-struct_sheet_order.range_id_2 } \\
\text {-struct_sheet_order.offset } \\
\text { _struct_sheet_order.sense }\end{array}$} \\
\hline A & 1_A & $2 \_\mathrm{A}$ & +1 & anti-parallel \\
\hline A & 2_A & 3_A & +1 & parallel \\
\hline B & $1{ }_{B}$ & 2_B & +1 & anti-parallel \\
\hline B & $10 \_B$ & $11 \_B$ & +1 & anti-parallel \\
\hline B & 2_B & 3_B & +1 & anti-parallel \\
\hline B & 3_B & 4 & +1 & anti-parallel \\
\hline B & 4_B & 5 _8 & +1 & parallel \\
\hline & 5_B & 6_B & +1 & parallel \\
\hline B & 6_B & 7_B & +1 & parallel \\
\hline B & 7_B & 8_B & & parallel \\
\hline B & $8 \_B$ & 9_B & & parallel \\
\hline B & 9_B & $10 \_B$ & & parallel \\
\hline
\end{tabular}

10op_

_struct_sheet_range.sheet_id

_struct_sheet_range.id

_struct_sheet_range.beg_label_comp_id _struct_sheet_range.beg_label_asym_id

_struct_sheet_range.beg_auth_seq_id

-struct_sheet_range.end_label_comp_id

_struct_sheet_range.end_label_asym_id

_struct_sheet_range.end_auth_seq_id

_struct_sheet_range.beg_label_seq_id

-struct_sheet_range.end_label_seq_id

A 1 _A LEU *6 6 THR * 10

A 6 T 6

A 2_A GLY * $\begin{array}{llll}13 & \text { MET * } & 16 \\ 13 & & 16\end{array}$

A 3_A VAL * 57 ALA * 60

57 a 60

B 1_B MET * 16 PRO * 21

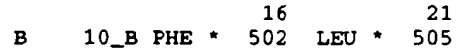

$502+505$

B 11 B MET * 510 GLN * 514

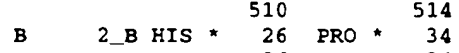

B 3 B TYR * 26 PRO 34

B 3_B TYR * 96 PRO * 102

B 4_B VAL * 142 SER * 147

B $142+147$

109 TYR * 116

B 6_B THR * 193 GLU * 1169

$\begin{array}{rl}193 & 199 \\ 220 & 226\end{array}$

B 7_B ARG * 220 SER * 226

B 8_B GLN * 318 ASN * 324

B 9_B GLY * $\begin{aligned} & 318 \\ & 417\end{aligned}$ PHE * $\begin{array}{r}324 \\ 423\end{array}$

Fig. 5 (cont.)
Table 4. pdb2cif conversion times (s) on an SGI R8000 Power Indigo-2

\begin{tabular}{lccc} 
& \multicolumn{3}{c}{ Size in characters $(\times 1000)$} \\
PDB entry & PDB & mmCIF & Conversion time (s) \\
4INS & 117 & 130 & 2.7 \\
ICTJ & 170 & 179 & 2.7 \\
2ACE & 393 & 433 & 7.3 \\
4HIR & 1753 & 1896 & 28.8
\end{tabular}

ATOM/HETATM records in PDB entries conforming to the 1996 PDB format have a field for a segment ID. The field is mapped to the mmCIF data item _atom_site.auth_asym_id, but the data type used in the dictionary does not permit embedded blanks, which may occur in the field. The problem is side-stepped for totally blank fields by mapping them to a period. Nonblank segment IDs are presented in the mmCIF data-set in quotation marks, e.g. as ' $\mathrm{VH} 1$ ', but, strictly speaking, if the rule in the mmCIF dictionary for this data item is not relaxed, the embedded blank should be replaced to make a valid $\mathrm{mmCIF}$ data-set.

It must be noted, even though the documentation of the program includes a partial concordance between PDB format and $\mathrm{mmCIF}$, the program itself is not table driven. At present the relationship between PDB format and $\mathrm{mmCIF}$ is too complex to be handled by use of a table. However, it may be helpful in understanding the discussion that follows to refer to the extract from the concordance given in Fig. 7. The full concordance can be found at http://ndbserver.rutgers.edu/ NDB/mmcif/software/pdb2cif/concord.html.

One of the most challenging parts of the conversion done by pdb2cif is the identification of chemical entities. pdb2cif does this by scanning SEQRES and ATOM PDB records for sequence homology indicating homologous chains and therefore equivalence as chemical entities. Doubtful cases are reported by warning comments in the mmCIF output. In mmCIF, an appropriate entity must be assigned to each unique structural element in the asymmetric unit. This includes polypeptide chains, polynucleotide chains, solvent, counter ions and other discrete chemical components such as inhibitors. If the same chemical entity appears more than once it must be given the same entity identification. This differs from PDB format in which chains are not explicitly associated with particular chemical entities. Let us first consider the handling of heterogens.

In the PDB format there is effectively an explicit identification of heterogeneous molecular entities by means of PDB FORMUL records. Each heterogen that is not integrated into

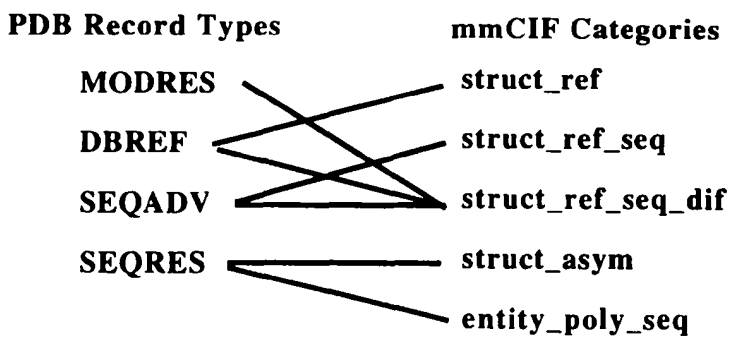

Fig. 6. Mapping of PDB primary structure section records to $\mathrm{mmCIF}$ categories. 
the backbone of a chain has a component number in columns 9-10 of the associated FORMUL record that may be used as a value for the mmCIF _entity.id Within an entry this number uniquely identifies the particular heterogen as a chemical entity. Alternatively, the PDB threc-letter heterogen ID (HetID) in columns 13-15 of the FORMUL record and columns 8-10 of the HET record could equally well be used to identify uniquely the entity for a heterogen. While the HetID has the singular advantage of being an identifier with global meaning valid for all PDB entries, the mmCIF token _struct_asym.id can be used to hold the HetID just as well. Therefore, for heterogens, we assign the FORMUL component number as the mmCIF_entity.id for heterogens, so that the PDB assignments will not be lost.

The identification of molecular entities for macromolecular chains is more complex and requires the use of implicit, rather than explicit, information from the PDB entry. Consider the sequence and heterogen information from PDB cntry 4INS (Baker et al., 1988) given in Fig. 8(a). There are four polypeptide chains $(A, B, C$ and $D)$, two zinc ions and 350 solvent molecules. On inspection of the sequences, it is clear that chains $A$ and $C$ are identical and chains $B$ and $D$ are identical. The program pdb2cif makes the same inspection by representing each residue by a single letter, converting each chain sequence into a character string and then performing substring matching to identify the chains that agree. The program insists on an exact match to declare two chains to be the same chemical entity, but warns of chains that show a match of more than $85 \%$ and less than $100 \%$ of the sequence. In the $\mathrm{mmCIF}$ produced by pdb2cif the entity category is used to report the distinct entity types and the struct_asym category is used to report the entity assigned to each chain or heterogen in the asymmetric unit. The chem_comp category is used to hold the chemical information. Note that, in order to satisfy mmCIF requirements for complete information about all the chemical components used, we list the amino acids as well as the heterogens. The resulting entity assignments made by $p d b 2 c i f$ are shown in Fig. $8(b)$.

Subsequence matching is then used to assign positions within the mmCIF entity_poly_seq table to each residue in the atom list. On each matching pass, an attempt is made to match the entire length of the remaining unmatched sequence and then the matching window is reduced by factors of the square root of two until we are working with a sequence fragment of length 16 or less, and then the window is reduced one residue at a time. The PDB ATOM list does not directly associate a residue with a position on the chain sequence. since the residue numbering used in the PDB ATOM list can have deletions, insertions, or be numbered in any arbitrary manner (even backwards or with negative numbers) prescribed by the author. Therefore, the residuc numbers in the PDB ATOM list cannot be used for this assignment. However, pdb2cif does issue a warning message if the sequence matches the implicit ATOM list sequence for less than $90 \%$ of a chain. In the case of NMR entries, a crosscomparison is also made between the implicit sequences of each of the models and a warning is issued if any mismatches are found. Consider PDB entry 1CWP (cowpea chlorotic mottle virus) (Speir et al., 1995). The sequence information in the SEQRES records shown in Fig. $9(a)$ implicitly defines a single entity for chains $A, B$ and $C$, starting MET, SER, THR, but the ATOM list starts with residue 42 . pdb2cif correctly makes the necessary sequence number assignments despite only $78 \%$ homology for chain $A$. This information is analyzed

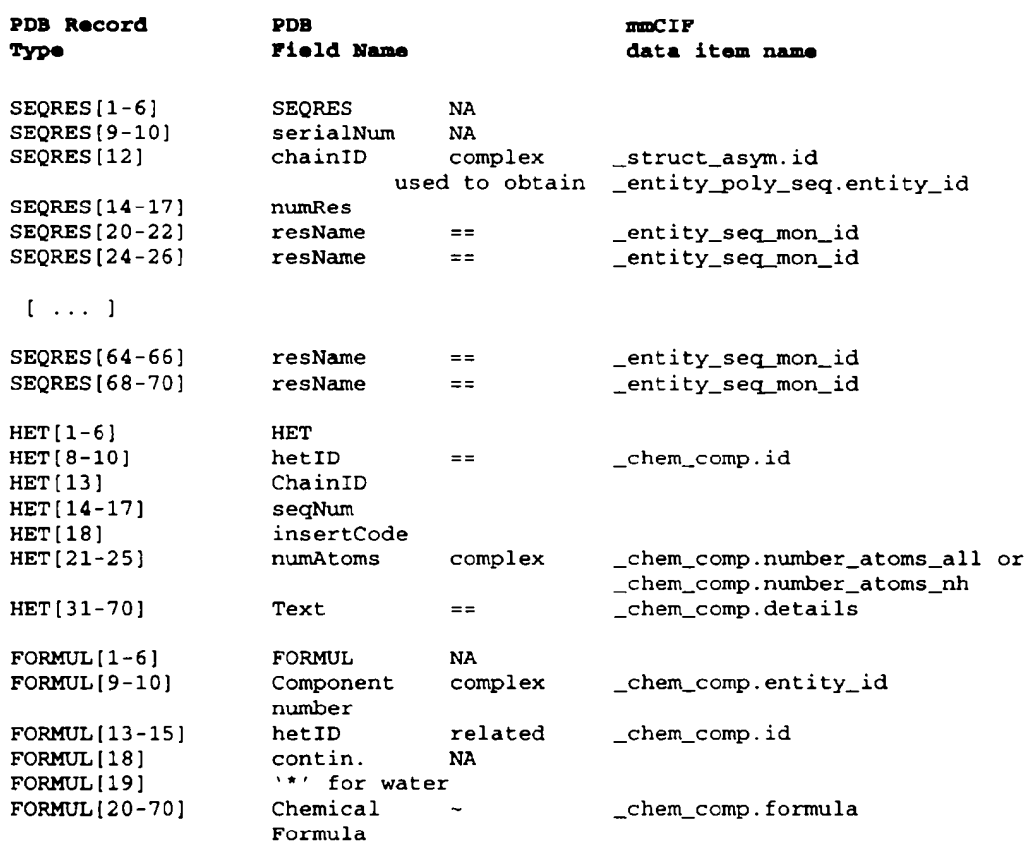

Fig. 7. Extract from the partial concordance of PDB format and mmCIF. The concordance shows some of the information needed to understand the mapping from PDB SEQRES records to mmCIF entities. (The notation '==' means 'equivalent to": ' $\sim$ ' means 'approximately equivalent to'; 'complex' means that a complex transition is involved; 'related' means that there is a relationship; and 'NA' means 'not applicable'.) 
to find one entity for polypeptide chains $A, B$ and $C$, a second entity for polynucleotide chains $D$ and $F$, and a third entity for polynucleotide chain $E$. When the first entity sequence is matched to the ATOM list, only $78 \%$ homology is found for chain $A$, and $86 \%$ for chains $B$ and $C$. The entity/sequence assignments (Fig. 9b) are then applied to the ATOM list without use of the author-assigned residue numbers or insertion codes, but purely from sequence homology. The result, shown in Fig. $9(c)$ is the same identification as made by the authors of 1CWP.

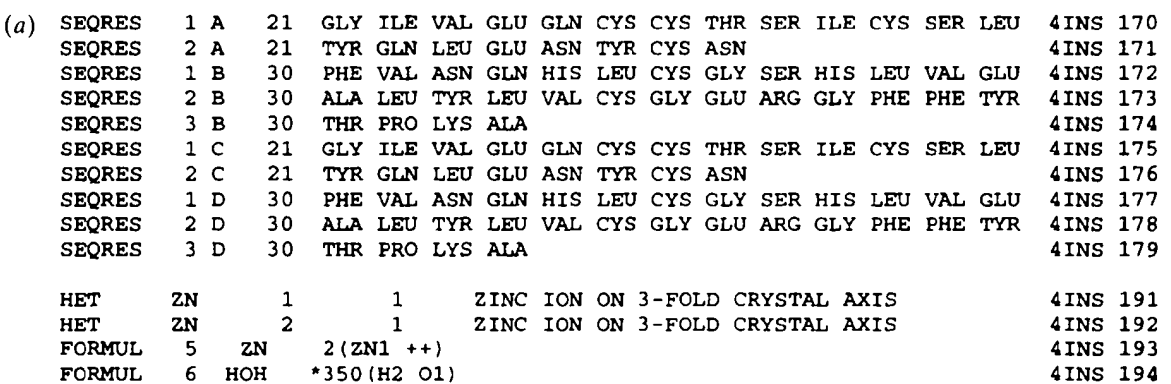

(b) loop

_entity.id

entity.type

-entity.details

1 polymer

; Protein chain: A, C

2 polymer

; Protein chain: B. D

5 non-polymer 'het group $2 \mathrm{~N}$ '

water ' $\mathrm{HOH}$ '

1oop_

struct_asym.entity_id

struct_asym.id

$1 \bar{A}$

2 B

$1 \mathrm{C}$

$2 \mathrm{D}$

$\begin{array}{lr}5 & \mathrm{ZN} \\ 6 & \mathrm{HOH}\end{array}$

loop_

_chem_comp.id

_chen_comp.mon_nstd_flag

chem_comp. formula

chem_comp.name

ZN no

; $2(2 \mathrm{~N} 1++)$

; ZINC ION ON 3-FOLD CRYSTAL AXIS

HOH no

: $350(\mathrm{H} 2 \mathrm{O})$

;

ALA yes ' $\mathrm{C} 3 \mathrm{H} 7 \mathrm{N1} \mathrm{O} 2$ '

ARG yes ' $\mathrm{C} 6 \mathrm{H}_{14} \mathrm{N4} \mathrm{O}_{2}$ '

ASN yes ' $\mathrm{C} 4 \mathrm{H} 8 \mathrm{N2} \mathrm{O}^{\prime}$

CYS yes ' $\mathrm{C} 3 \mathrm{H} 7 \mathrm{N1}$ O2 $\mathrm{S} 1$

GLN yes 'C5 H10 N2 O3'

GLU yes ' $\mathrm{C} 5 \mathrm{H} 9 \mathrm{~N} 1 \mathrm{O}^{\circ}$

GLY yes ' $\mathrm{C} 2$ H5 N1 O2'

HIS yes 'C6 H9 N3 O2

ILE Yes ' $\mathrm{C} 6 \mathrm{H} 13$ N1 O2

LEU yes ' $\mathrm{C} 6 \mathrm{H} 13 \mathrm{~N} 1 \mathrm{O} 2$

LYS yes ' $\mathrm{C} 6 \mathrm{H} 14$ N2 $\mathrm{O} 2$

PHE yes 'C9 $\mathrm{H} 11 \mathrm{~N} 1 \mathrm{O} 2$ '

PRO Yes ' $\mathrm{C} 5 \mathrm{H} 9 \mathrm{~N} 1 \mathrm{O} 2$

SER yes ' $\mathrm{C} 3 \mathrm{H} 7 \mathrm{N1} \mathrm{O} 3$

THR Yes ' $\mathrm{C} 4 \mathrm{H} 9 \mathrm{~N} 1 \mathrm{O}$

TYR yes ' $\mathrm{C} 9 \mathrm{H} 11 \mathrm{~N} 1 \mathrm{O}^{\prime}$ '

VAL yes ' $\mathrm{C} 5 \mathrm{H} 11 \mathrm{~N} 1 \mathrm{O} 2$

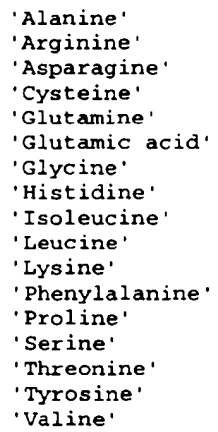

Fig. 8. (a) Sequence and heterogen information from PDB entry 4INS. (b) Entity assignments made by pdb2cif for PDB entry 4INS. 
(a) SEQRES 1 A 190 MET SER THR VAL GLY THR GLY LYS LEU THR ARG ALA GLN 1CWP 114

SEQRES 2 A 190 ARG ARG ALA ALA ALA ARG LYS ASN LYS ARG ASN THR ARG 1CWP 115

SEQRES 3 A 190 VAL VAL GLN PRO VAL ILE VAL GLU PRO ILE ALA SER GLY 1CWP 116

$\begin{array}{lllllll}\text { SEQRES } & 4 & \text { A } & 190 & \text { GLN GLY LYS ALA ILE LYS ALA TRP THR GLY TYR SER VAL } & \text { 1CWP } 117\end{array}$

SEQRES 5 A 190 SER LYS TRP THR ALA SER CYS ALA ALA ALA GLU ALA LYS 1CWP 118

SEQRES 6 A 190 VAL THR SER ALA ILE THR ILE SER LEU PRO ASN GLU LEU 1CWF 119

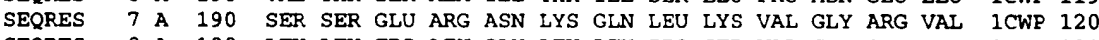

$\begin{array}{llllllll}\text { SEQRES } & 8 & \text { A } & 190 & \text { LEU LEU TRP LEU GLY LEU LEU PRO SER VAL SER GLY THR 1CWP } 121\end{array}$

SEQRES 9 A 190 VAL LYS SER CYS VAL THR GLU THR GLN THR THR ALA ALA 1CWP 122

SEQRES 10 A 190 ALA SER PHE GLN VAL ALA LEU ALA VAL ALA ASP ASN SER 1CWP 123

SEQRES 11 A 190 LYS ASP VAL VAL ALA ALA MET TYR PRO GLU ALA PHE LYS 1CWP 124

SEQRES 12 A 190 GLY ILE THR LEU GLU GLN LEU ALA ALA ASP LEU THR ILE 1CWP 125

SEQRES 13 A 190 TYR LEU TYR SER SER ALA ALA LEU THR GLU GLY ASP VAL 1CWP 126

SEQRES 14 A 190 ILE VAL HIS LEU GLU VAL GLU HIS VAL ARG PRO THR PHE 1CWP 127

SEQRES $15 \mathrm{~A} 190$ ASP ASP SER PHE THR PRO VAL TYR

SEQRES I B 190 MET SER THR VAL GLY THR GLY LYS LEU THR ARG ALA GLN 1CWP 129

SEQRES 2 B 190 ARG ARG ALA ALA ALA ARG LYS ASN LYS ARG ASN THR ARG 1CWP 130

[ ... portions of chains $B$ and $C$ omitted here ...]

SEQRES 13 C 190 TYR LEU TYR SER SER ALA ALA LEU THR GLU GLY ASP VAL 1CWP 156

SEQRES $14 \mathrm{C} 190$ ILE VAL HIS LEU GLU VAL GLU HIS VAL ARG PRO THR PHE 1 CWP 157

SEQRES $15 \mathrm{C} 190$ ASP ASP SER PHE THR PRO VAL TYR

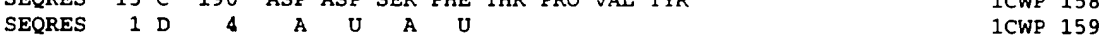

$\begin{array}{lllllll}\text { SEQRES } & 1 & \text { E } & 2 & \text { A } & U & \text { 1CWP } 160\end{array}$

$\begin{array}{llllllllll}\text { SEQRES } & 1 & \text { F } & 4 & \text { A } & \text { U } & \text { A } & \text { U } & \text { 1CWP } & 161\end{array}$

(b) loop

_entity_poly_seq.entity_id

_entity_poly_seq.num

_entity_poly_seq.mon_id

\begin{tabular}{|c|c|c|c|c|c|c|c|c|c|c|c|c|c|}
\hline 1 & $1 \mathrm{MET}$ & 1 & 2 & SER & 1 & 3 & THR & 1 & 4 & VAL & 1 & 5 & GLY \\
\hline 1 & 6 THR & 1 & 7 & GLY & 1 & 8 & LYS & 1 & 9 & LEU & 1 & 10 & THR \\
\hline 1 & 11 ARG & 1 & 12 & ALA & 1 & 13 & GLN & 1 & 14 & ARG & 1 & 15 & ARG \\
\hline 1 & $16 \mathrm{ALA}$ & 1 & 17 & $A L A$ & 1 & 18 & ALA & 1 & 19 & ARG & 1 & 20 & LYS \\
\hline 1 & 21 ASN & 1 & 22 & LYS & 1 & 23 & $A R G$ & 1 & 24 & ASN & 1 & 25 & THR \\
\hline 1 & 26 ARG & 1 & 27 & VAL & 1 & 28 & VAL & 1 & 29 & GLN & 1 & 30 & PRO \\
\hline 1 & 31 VAL & 1 & 32 & ILE & 1 & 33 & VAL & 1 & 34 & GLU & 1 & 35 & PRO \\
\hline 1 & 36 ILE & 1 & 37 & ALA & 1 & 38 & SER & 1 & 39 & GLY & 1 & 40 & GLN \\
\hline 1 & $41 \mathrm{GLY}$ & 1 & 42 & LYS & 1 & 43 & ALA & 1 & 44 & ILE & 1 & 45 & LYS \\
\hline 1 & 46 ALA & 1 & 47 & TRP & 1 & 48 & THR & 1 & 49 & GLY & 1 & 50 & TYR \\
\hline 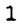 & 51 SER & 1 & 52 & VAL & 1 & 53 & SER & 1 & 54 & LYS & 1 & 55 & TRP \\
\hline
\end{tabular}

[ ... portions of entity 1 sequence omitted ... ]

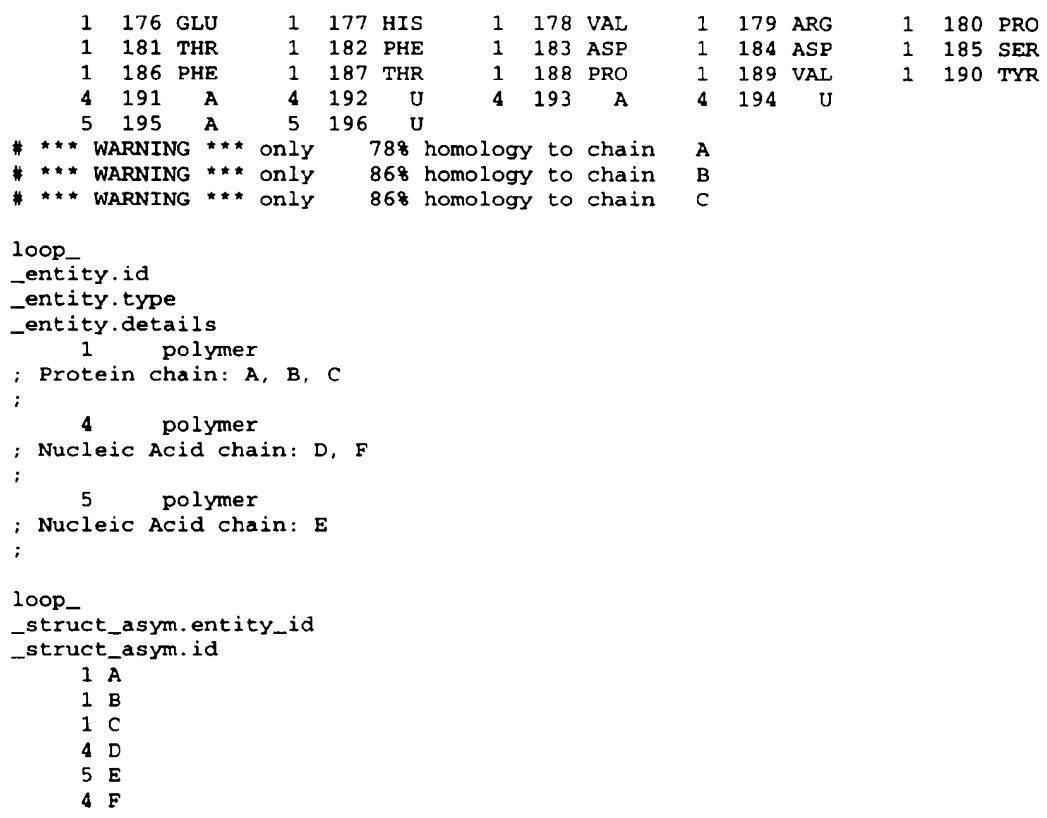

Fig. 9. (a) Sequence information from PDB entry 1CWP. (b) Entity assignments made by pdb2cif for PDB entry 1CWP. (c) Entity assignments in the atom_site table made by pdb2cif for PDB entry 1 CWP. 
The program accepts all current PDB record types. Figs. $10(a)$ and $10(b)$ show examples for the DBREF and ANISOU PDB record types from the PDB entry 1CTJ (cytochrome c6) (Frazao et al., 1995). pdb2cif inserts the necessary tags and values into the atom_site table, but uses a different ordering, as shown in Fig. 10(c). Also, note the change in scaling, because the values for anisotropic $U$ in mmCIF are not multiplied by 10000 as in PDB entries.

The organization of the atom_site records into lines was dictated by the limit of 80 characters per line in mmCIF, a desire to keep related information together and organized into columns that could easily be scanned by eye. It would have made an equally valid $\mathrm{mmCIF}$ data-set to have removed most of the white space and presented the three lines of data which are the first row of the table as:

1 . ATOM N N GLU * 1 A $4.127 \quad 26.179 \quad-7.903$ $\begin{array}{lllllllllll}0.49 & 57.53 & \text {. } & 1 & 1 & 0.9336 & 0.0004 & 0.2737 & 0.7394\end{array}$ 0.27710 .4591

\section{Dealing with blanks}

A PDB entry may have many blank fields and omitted records, but mmCIF format does not permit blank or skipped fields. This restriction in mmCIF is necessary in order to retain the correct alignment of the name-value mapping between the column headings and the values within tables. For example, in the ATOM records of 1CTJ above, the chain identifier, the insertion code and footnote fields are blank. In most cases, pdb2cif translates a blank field in PDB format to a period, to denote an intentionally blank field. In some cases, question marks are used instead of periods in, for example, some fields in citations, because there is a possibility that some of the information could be filled in from other sources (e.g. _citation.journal_issue). Blank insertion codes are ignored rather than converted. since pdb2cif appends the insertion code to the residue number to form _atom_site.auth_seq_id. There is no possibility of unintentional duplications in this field in recent PDB entries, since the PDB does not use numerical insertion codes. However, it is possible that some old PDB entries might contain numeric insertion codes. In those few cases, it is possible that residue ' 9 ' with insertion code ' 2 ' might be confused with residue ' 92 '. When the PDB converts its older entries to the current format, any numeric insertion codes will be changed to alphabetic characters.

The most difficult question of blank fields arises from blank chain identifiers in PDB entries. The PDB uses a blank as the chain identifier in almost all entries with only one chain. In this case, a quoted blank or a question mark as the mmCIF translation of the blank PDB chain identifier might have the wrong connotation. Therefore, except when translating the chain identifiers for heterogens in a structure with multiple chains, we replace a blank chain identifier with an asterisk. An asterisk is not a special character in $\mathrm{MmCIF}$, but is a character that is never used in PDB entries for a chain identifier. This provides a valid chain identifier in the $\mathrm{mmCIF}$ data-set while preserving the information that the original chain identifier in the PDB entry was blank. Therefore, atom_site records for heterogens in which the PDB chain identifier is blank are given as a period for atom_site.asym_id unless the PDB entry has only one chain that had a blank chain identifier in the PDB entry. This avoids any implications about chain assignments for heterogens in a PDB entry with multiple chains for which the PDB entry did not make any chain assignment.

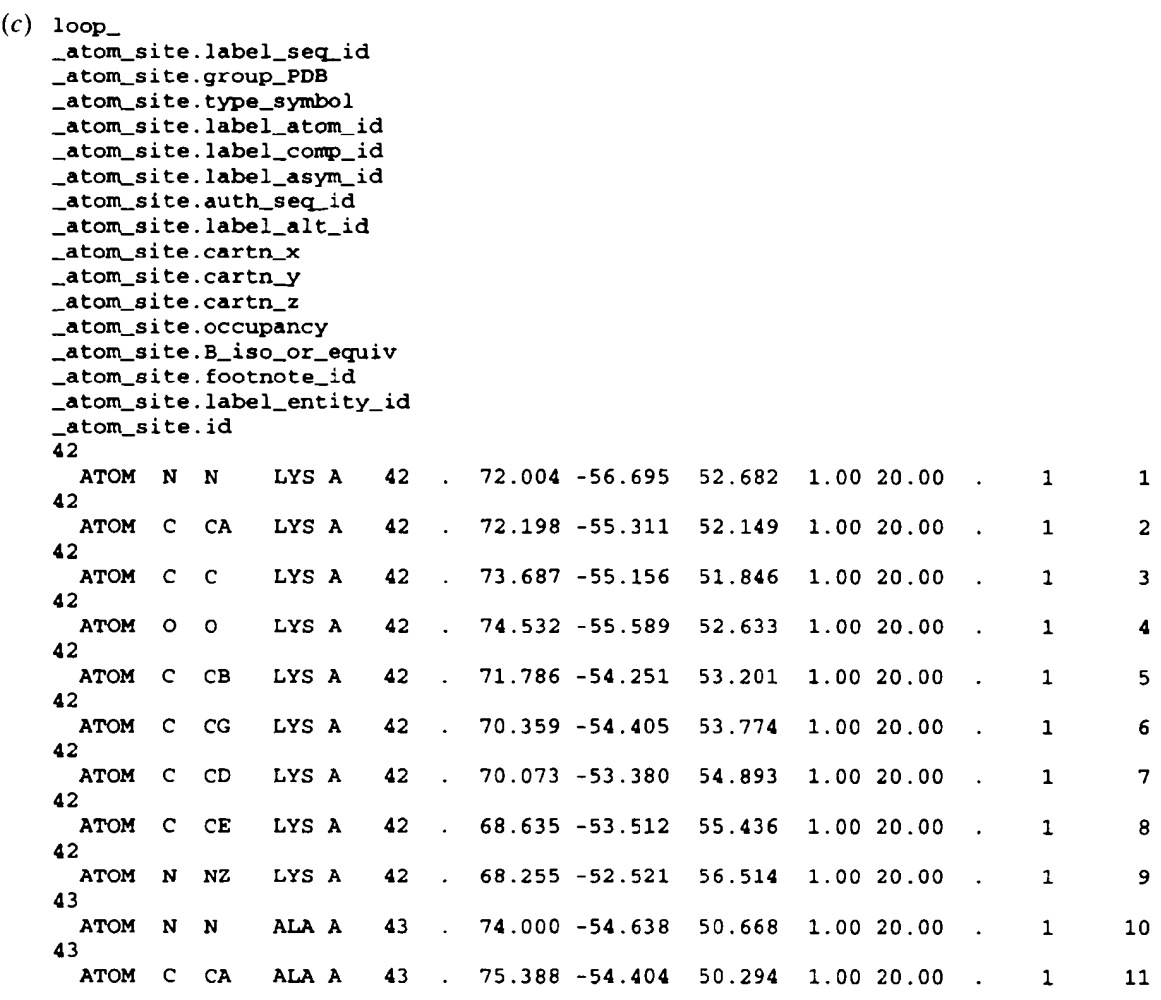

Fig. 9 (cont.) 
$\begin{array}{lllllllll}\text { (a) DBREF } & 1 \mathrm{CTJ} & 1 & 89 & \text { SWS } & \text { Q09099 } & \text { CYC6_MONBR } & 1 & 89\end{array}$

is converted into two tables by pdb2cif:

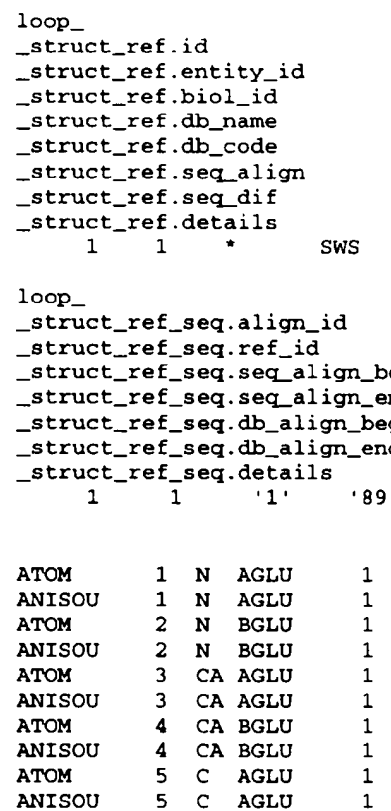

$\cdot 1 \cdot .89^{\prime}$.

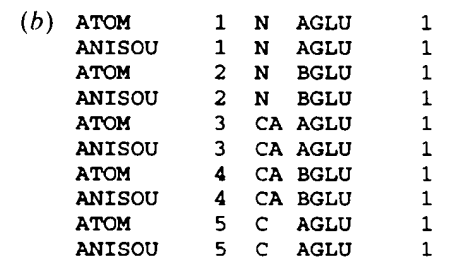

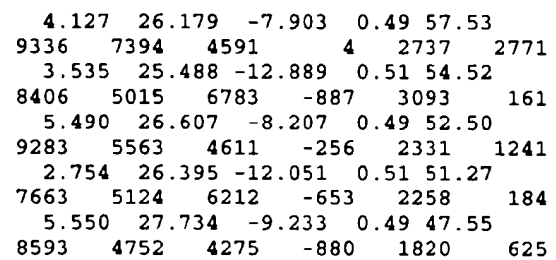

(c) loop

_atom_site.Iabel_seq_id

atom_site.auth_asym_id

atom_site.group_PDB

-atom_site.type_symbol

atom_site.label_atom_id

_atom_site. label_comp_id

_atom_site.label_asym_id

_atom_site.auth_seq_id

-atom_site.label_alt_id

atom_site.cartn_x

_atom_site.cartn_y

-atom_site.cartn_z

-atom_site.occupancy

atom_site. B_iso_or_equiv

atom site. footnote id

atom_site.label_entity_id

_atom_site.id

atom site aniso U[1] [1]

atom site.aniso U[1] [2]

_atom_site.aniso_U[1] [3]

_atom_site.aniso_U[2] [2]

-atom_site.aniso_U[2] [3]

-atom_site.aniso_U [3] [3]

1

Aтом N N GLU * $\quad \begin{array}{llllllllll}1 & \text { A } & 4.127 & 26.179 & -7.903 & 0.49 & 57.53 & \text {. } & & 1\end{array}$

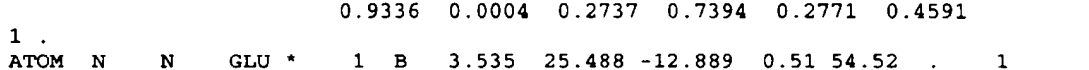

$\begin{array}{llllllllll}1.8406 & -0.0887 & 0.3093 & 0.5015 & 0.0161 & 0.6783 & \end{array}$

$\begin{array}{llccccccccc}\text { ATOM C CA GLU * } & 1 & \text { A } & 5.490 & 26.607 & -8.207 & 0.49 & 52.50 & . & 1 & 3 \\ 0.9283 & -0.0256 & 0.2331 & 0.5563 & 0.1241 & 0.4611 & & \end{array}$

$\begin{array}{lllllllllllll}1 & \text { ATOM C CA GLU * } & 1 & \text { B } & 2.754 & 26.395 & -12.051 & 0.51 & 51.27 & 1 & 4\end{array}$

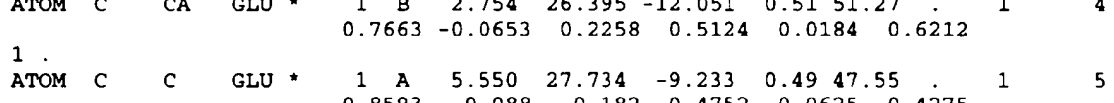

Fig. 10. (a) DBREF information from 1CTJ translated to the struct_ref and struct_ref_seq tables. (b) Anisotropic temperature factors from PDB entry 1CTJ. (c) Translation of anisotropic temperature factors from PDB entry 1CTJ to appropriate values in the atom_site table. Note the change in scaling. 


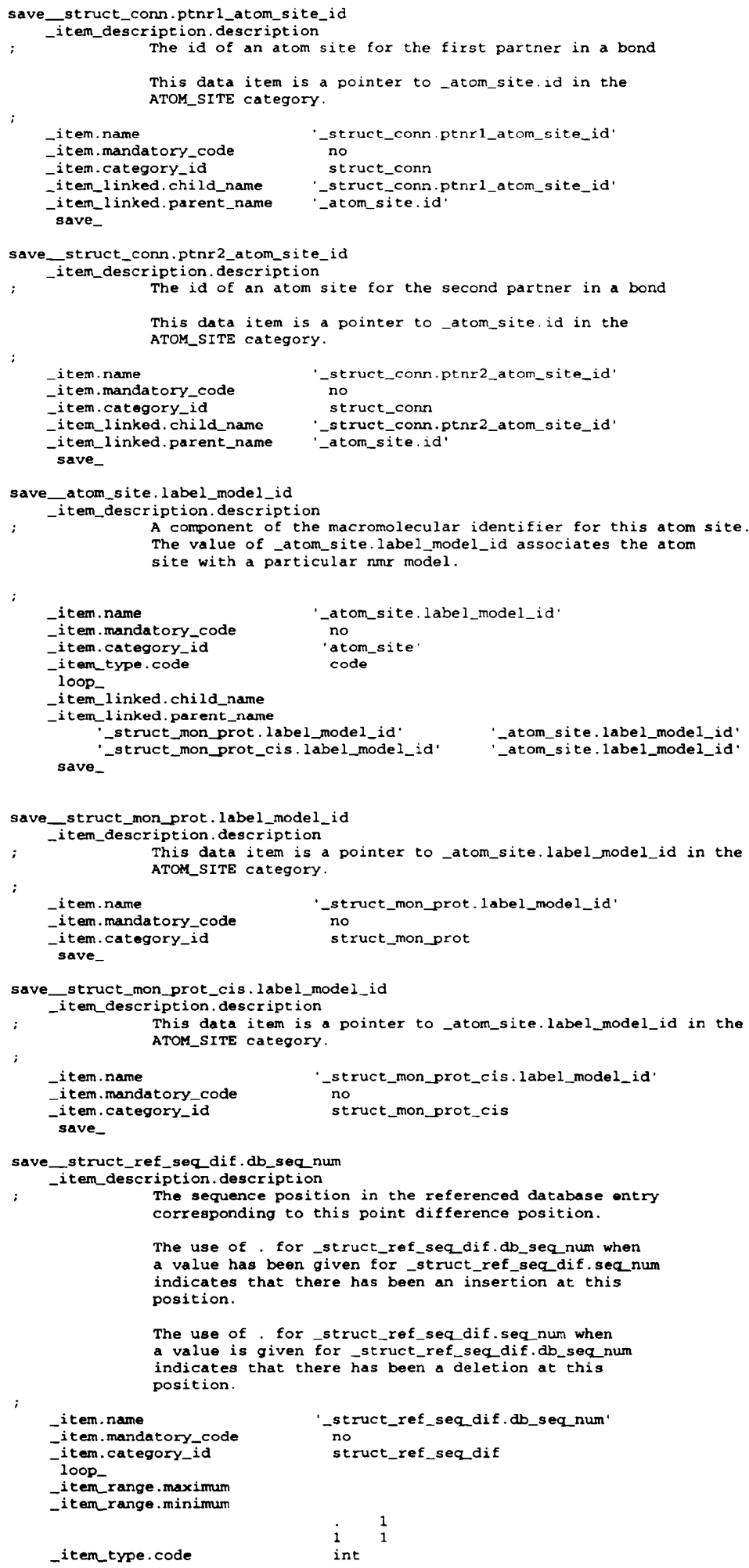

save_

Fig. 11. Definitions used by pdb2cif which are not in the mmCIF dictionary. 


\section{7. $\mathrm{mmCIF}$ compliance}

The program pdb2cif can translate a PDB entry into a data-set that is substantially compliant with the mmCIF dictionary. although careful checking of the results is suggested. This version is intended to produce mmCIF files conforming to mmCIF version 1.0.00 and above. Full compliance is not possible in some areas. In particular, most of the values used for _exptl.method, and some of the values used for _struct_conf_type.id do not conform to the enumerations in the dictionary. Full compliance would require agreement between the PDB and COMCIFS (the IUCr committee that oversees the CIF dictionaries) on equivalent lists of values. In addition, the PDB has relcased some entries with truncated author lists, using 'ET AL.' to indicate the missing authors. This practice does not conform to $\mathrm{mmCIF}$ requirements and pdb2cif does not have access to the information necessary to complete the list of authors.

In order to translate PDB records completely without information loss, pdb2cif uses a few tokens that are not in the dictionary. If strict dictionary validation is done, the definitions shown in Fig. 11 would have to be appended to the mmCIF dictionary for validation of pdb2cif output.

\section{Future plans}

Plans call for an extension of the parsing of the internal fields of COMPND and SOURCE and of the newer, more structured remarks (Protein Data Bank, 1996) and compliance with the mmCIF dictionary as it evolves. Ultimately, our goal is to convert from PDB format to $\mathrm{mmCIF}$ in sufficient detail as to extract all information for which $\mathrm{mmCIF}$ tokens exist and for which information was provided in an entry, while preserving the names and relationships that existed in the PDB entry. In this way, all records of the original entry can be reconstructed from the new mmCIF data-set.

\section{Distribution}

The latest version of this software is available at any of the following WWW servers:

http://www.sdsc.edu/pb/pdb2cif/pdb2cif

http://ndbserver.rutgers.edu/NBD/mmcif/software

http://www.ebi.ac.uk/NDB/mmcif/software

http://ndbserver.nibh.go.jp/NDB/mmcif/software

http://www.iucr.org/iucr-top/cif/software/pdb2cif

pdb2cif is distributed as pdb2cif.cshar. Z, a compressed $\mathrm{C}$-shell self-extracting archive. The structure of this file permits automatic unpacking on Unix systems using the $\mathrm{C}$ shell, csh, but, unlike the more commonly used 'shar' format, also permits unpacking with a text editor. A pdb2cif . shar. Z version is also available.

If an mmCIF data-set produced from a particular PDB entry is required, the 3DB browser (Abola et al., 1996) available at http://www.pdb.bnl.gov has an interface to pdb2cif as an output option. Alternatively, the MOOSE database
(Shindyalov et al., 1995) available at http:/www.sdsc.edu/ moose also has an option to display the mmCIF version of any PDB-formatted file. For further information, e-mail yaya@ bernstcin-plus-sons.com.

This work was supported in part by US NSF, PHS. NIH, NCRR. NIGMS, NLM and DOE under contract DE-AC02$76 \mathrm{CH} 00016$ (for FCB), US NSF grant no. BIR 9310154 (for PEB), and the IUCr (for HJB).

\section{References}

Abola. E. E.. Prilusky, J., Manning. N. O. \& Sussman. J. L. (1996). Acta Cryst. A52 Supplement, C-586.

Baker, E. N., Blundell, T. L., Cutficld, J. F., Cutfield, S. M., Dodson, E. J., Dodson. G. G.. Crowfoot Hodgkin. D. M.. Hubbard, R. E. Isaacs, N. W., Reynolds, C. D., Sakabe, K., Sakabe, N. \& Vijayan. N. M. (1988). Philos. Trans. R. Soc. London, 319, 369-456.

Berman, H. M. \& Westbrook. J. D. (1994). A Gentle Introduction to One Working Alternative DDI, for Macromolecular Structure. In European Macromolecular Crystallographic Information $(\mathrm{mmCIF})$ Workshop, edited by S. D. Wodak. Free University of Brussels, European Commission.

Bernstein. F. C., Koetzle, T. F., Williams, G. J. B., Mever, E. F. Jr, Brice. M. D., Rodgers, J. R., Kennard, O., Shimanouchi, 'T. \& Tasumi, M. (1977). J. Mol. Biol. 112, 535-542.

Bourne, P. E., Berman, H. M.. McMahon. B., Watenpaugh, K. D. Westbrook, J. \& Fitzgerald, P. M. D. (1997). Methods Enzymol. 277, 571-590.

Codd, E. F. (1970). Commun. ACM, 13, 377-387.

Codd, E. F. (1972). Data Base Systems, edited by R. Rustin, pp. 33-64. Englewood Cliffs, NJ: Prentice-Hall.

Fitzgerald, P. M. D., Berman, H. M., Bourne, P. E., McMahon, B. Watenpaugh, K. \& Westbrook, J. (1996). Acta Cryst. A52 Supplement, C-576.

Frazao, C., Soares, C. M.. Carrondo, M. A., Pohl. E., Dauter, Z.. Wilson, K. S., Hervas, M., Navarro, J. A., De La Rosa, M. A. \& Sheldrick, G. M. (1995). Structure (London), 3, 1159-1169.

Hall, S. R. (1991). J. Chem. Inf. Comput. Sci. 31, 326-333.

Hall, S. R. \& Spadaccini. N. (1994). J. Chem. Inf. Comput. Sci. 34, 505-508 (see http://www.crystal.uwa.edu.au/cc_star.html).

Kernighan, B. W. \& Ritchie, D. M. (1977). The M4 Macro Processor. Murray Hill, NJ: Bell Laboratories.

Protein Data Bank (1996). The Protein Data Bank Contents Guide: Atomic Coordinate Entry Format Description, Version 2.1, http:// www.pdb.bnl.gov/format.doc/format_home.html.

Raves. M. L., Harel. M., Pang, Y.-P., Silman, I., Kozikowski, A. P. \& Sussman, J. L. (1977). Nat. Struct. Biol. 4. 57-63.

Shindyalov, I. N., Cooper, J., Chang, W. \& Bourne, P. E. (1995). Proceedings of the 28th Hawaii International Conference on System Sciences, pp. 207-217. Los Alamitos, CA: IEEE Computer Society Press.

Speir, J. A., Munshi, S., Wang, G.. Timothy, S., Baker, J. E. \& Johnson. J. E. (1995). Structure (London), 3, 63-78.

Westbrook. J. \& Hall. S. R. (1995). A Dictionary Description Language for Macromolecular Structure, Draft DDL V 2.1.0, IUCr COMCIFS, Chester, England. Available from http://ndbserver.rutgers.edu/ $\mathrm{NDB} / \mathrm{mmcif} / \mathrm{ddl} /$ index.html. 